\title{
Estudio comparativo de los tres papeles de filtro en los ensayos UMELISA para el tamizaje neonatal de hipotiroidismo congénito y fenilcetonuria.
}

\section{Artículo Original}

Amarilys Frómeta-Suárez, Neivis Marrero-González, Ernesto González-Reyes, Elena Lugo-Vallejo, Lianny Fernández-Fernández, Marina B. Doménech-Mylnikova.

Laboratorio de Tamizaje Neonatal. Centro de Inmunoensayo. Calle 134 y Ave.25, Apartado Postal 6653, Cubanacán, Playa, Ciudad Habana, Cuba.

\section{RESUMEN.}

Introducción. El tamizaje neonatal de Hipotiroidismo Congénito y otras enfermedades heredometabólicas comparten el hecho de que los analitos de interés son determinados en manchas de sangre seca colectada en papel de filtro. La concentración del analito depende de la forma en que son preparados los calibradores y de otras variables que están involucradas en este proceso; como el lote y el tipo de papel de filtro usado, la matriz de los calibradores, el porcentaje de hematocrito entre otros.

Material y métodos. Se evaluaron 4 controles preparados en sangre total y ajustados al $55 \%$ de hematocrito con diferentes niveles de concentración de TSH, T4 y Fenilalanina cada control fue dispensado en tarjetas de papel de filtro Whatman BFC 180, S\&S 2992 y 903 ${ }^{\mathrm{TM}}$, las concentraciones de cada analito se midieron utilizando los UMELISAS TSH y T4 Neonatal y en el UMTEST PKU. Se realizó un estudio de estabilidad durante 9 meses, para los controles conservados entre $2-8^{\circ} \mathrm{C}$, además fueron evaluadas 400 muestras de neonatos colectadas simultáneamente en papel de filtro 2992 y $903^{\mathrm{TM}}$.

Resultados y conclusiones. La aplicación de la prueba t para datos pareados demostró que no existen diferencias estadísticamente significativas entre los tres tipos de papel de filtro para un intervalo de confianza del $95 \%$ al no producir afectación en la precisión y exactitud de estos métodos. El porcentaje de pérdida de actividad de los 3 analitos estudiados fue inferior al 17\%. Los valores promedios de concentración en la distribución de TSH y Phe son ligeramente mayores en las muestras colectadas en papel $903^{\mathrm{TM}}$.

(Rev Biomed 2002; 13:241-247)

Palabras clave: Sangre impregnada en papel de filtro, UMELISA, tamizaje neonatal, hipotiroidismo congénito, fenilcetonuria.

\section{SUMMARY.}

Comparative studio of three filters papers in neonatal screening UMELISA assays for

Solicitud de sobretiros: M.Sc. Amarilys Frómeta-Suárez, Laboratorio de Tamizaje Neonatal. Centro de Inmunoensayo. Calle 134 y Ave.25, Apartado Postal 6653, Cubanacán, Playa, Ciudad Habana, Cuba. 


\section{A Frómeta-Suárez, N Marrero-González, E González-Reyes, E Lugo-Vallejo y col.}

phenylketonuria and congenital hypothyroidism. Introduction. Newborn screening for congenital hypothyroidism and other inherited metabolic diseases share the fact that the analytes are determined in dried blood spotted on filter paper. The analyte concentration depends on the way the calibrators are prepared and other variables involved in this process like the lots and types of filter paper used, the matrix of calibrators, and the percentage of hematocrit among others.

Material and methods. Four controls prepared in total blood and hematocrit adjusted to $55 \%$ with different concentrations of TSH, T4 and Phe were evaluated. Each control was spotted on filter paper cards, Whatman BFC 180, Schleicher and Schuell 2992 and $903^{\mathrm{TM}}$. The analyte concentration was determined by UMELISA TSH Neonatal, UMELISA T4 Neonatal and UMTEST PKU. A stability study on the controls stored at 2-8 C for 9 months was performed. In addition 400 samples of neonates collected on filter papers 2992 and $903^{\mathrm{TM}}$ were evaluated, at the same time.

Results and conclusions. Student's $t$ test showed there were no significant differences between the 3 types of filter papers $(\mathrm{p}<0.05)$ in that the precision and the accuracy of these methods weren't affected. The percentage of activity loss of analytes was less than $17 \%$. The media concentrations in the distributions of TSH and Phe were slightly higher in samples collected on filter paper $903^{\mathrm{TM}}$.

(Rev Biomed 2002; 13:241-247)

Key words: Blood spot, UMELISA, neonatal screening, congenital hypothyroidism, phenilketonuria.

\section{INTRODUCCION.}

Los programas de tamizaje neonatal de enfermedades tales como hipotiroidismo congénito, fenilcetonuria, galactosemia, fibrosis quística y otras enfermedades heredometabólicas, comparten el hecho de que los analitos o el analito de interés son determinados en manchas de sangre seca colectadas en papel de filtro por punción de talón, debido a que la detección clínica de estas patologías en la etapa neonatal es casi imposible ya que sus síntomas son muy subjetivos y escasos (1). La facilidad de conservación de muestras testigos por largos períodos, aún si se dispone de escasa capacidad de refrigeración (2), la no utilización de jeringuillas y tubos de ensayo ni la necesidad de un adiestramiento especial para la toma de muestra han hecho que esta sea la práctica más utilizada y difundida de colecta de muestra de sangre en neonatos a nivel internacional, para programas de pesquisa neonatal. En la actualidad el avance alcanzado en la obtención del ADN a partir de muestras colectadas en papel de filtro y su aplicación en la tecnología de la reacción en cadena de la polimerasa (PCR), ha incrementado la utilización de esta forma de toma de muestra para estudios genéticos en grupos de riesgo, pruebas de paternidad, investigaciones forenses y estudios poblacionales en general (3).

La concentración del analito medida en manchas de sangre neonatal depende de la forma en que son preparados los calibradores. Muchas variables están involucradas en este proceso; tales como el tipo y el lote del papel de filtro usado, la matriz de los calibradores, el porcentaje del hematocrito, el tamaño de la mancha de sangre, el uso de células rojas lisadas o intactas, el uso de anticoagulantes y bactericidas, las condiciones de almacenamiento y el secado, la preparación de referencia usada para establecer la concentración de los calibradores y el método de análisis usado (4).

En la selección del papel de filtro se debe considerar, la disponibilidad de un papel estándar de calidad suministrado por una fuente confiable y competitiva que garantice propiedades tales como: tipo de material, peso, densidad, $\mathrm{pH}$, porcentaje de humedad, consistencia y capacidad de absorción, además debe ajustarse a los procedimientos de toma de muestra estandarizados en cada país y una última característica pero no menos importante es que sus características funcionales estén dentro de los límites de aceptación para los ensayos destinados a la cuantificación de determinadas sustancias en fluidos biológicos colectados en papel de filtro (5).

Los tipos de papel de filtro más ampliamente

\section{Revista Biomédica}




\section{Papeles de filtro en los ensayos UMELISA.}

usados son el Whatman BFC 180 fabricado por Whatman International Ltd, Inglaterra, Schleicher \& Schuell 2992 utilizado fundamentalmente en Europa Occidental y Schleicher \& Schuell $903^{\mathrm{TM}}$ utilizado en USA y Australia ambos papeles son producidos por Schleicher \& Schuell Inc.GmbH, Alemania y Schleicher \& Schuell Inc, E.U.A respectivamente. En este trabajo se expone un estudio comparativo con estos tres tipos de papel, utilizados en los ensayos UMELISA $^{\circledR}$ destinados al diagnóstico neonatal de Hipotiroidismo Congénito y Fenilcetonuria.

\section{MATERIALES Y MÉTODOS.}

Preparación de los controles de tirotropina, tiroxina y fenilalanina en papel de filtro $S \& S$ 2992, S\&S 903 y Whatman BFC 180.

Las materias primas utilizadas para preparar los controles en sangre total, fueron suero humano y eritrocitos procedentes de donantes voluntarios, los cuales resultaron ser negativos a las pruebas de detección de Anti-VIH 1+2, Anti-VHC y HBsAg. -Controles de tirotropina (TSH)

Para la preparación de los controles de tirotropina (TSH) en sangre, se procedió al lavado de los eritrocitos con solución salina $0.9 \%$ y luego se provocó la lisis de los mismos. Se seleccionó un suero con bajas concentraciones de TSH (0 mUI/L).

\section{Controles de tiroxina (T4)}

Para la preparación de los controles de tiroxina en sangre se realizó el mismo procedimiento de lavado de los eritrocitos que los controles de TSH. El suero humano seleccionado fue liberado de T4 mediante el método de adsorción a carbón activado (6).

\section{Controles de fenilalanina (Phe)}

El procedimiento de preparación de los controles de Phe en sangre se realizó mediante el lavado de los eritrocitos con solución salina al $0.9 \%$, dichas células se mantuvieron intactas y se seleccionó un suero con bajas concentraciones de este aminoácido $(0.65 \mathrm{mg} /$ dL).

Idealmente un material de referencia debe estar compuesto por la misma matriz o una muy semejante a la de las muestras que van a ser analizadas. La matriz de los calibradores en sangre sobre papel de filtro debe ser muy parecida a la matriz de las muestras de sangre colectadas en los neonatos tanto como sea posible. El hematocrito de los neonatos es de 55-60\% en el primer día y entre $50-55 \%$ en los 5 días posteriores al nacimiento, por lo que se prefiere ajustar el hematocrito a 55\% (4). A la sangre total ajustada a este hematocrito se le añadió como bactericida azida sódica al $0.2 \%$ y luego se adicionaron las cantidades necesarias de tiroxina para obtener los controles de concentraciones 80; 100; 200; 300 nmol de T4/L de suero, así como, las cantidades necesarias de tirotropina para obtener controles de concentraciones 10, 20, 30, 60 mUI de TSH/L de sangre total. En el caso de Phe se adicionaron las cantidades necesarias para obtener los controles de concentraciones $2 ; 4$; 6; $10 \mathrm{mg}$ de Phe/dL de sangre total.

Se dispensaron $25 \mu \mathrm{L}$ de sangre de los controles en cada uno de los cuatro círculos definidos en las tarjetas de papel de filtro Schleicher \& Schuell 2992, Schleicher \& Schuell 903 ${ }^{\mathrm{TM}}$ y Whatman BFC 180 especialmente preparadas para este estudio.

Las tarjetas se colocaron en posición horizontal sobre soportes diseñados con este fin para evitar el contacto de las mismas con la superficie y se secaron durante 24 horas en un local con temperatura de $20^{\circ} \mathrm{C}$ a $25^{\circ} \mathrm{C}$ y humedad relativa $<50 \%$. Posteriormente se sellaron en sobres de PVC-papel de aluminio con sílica gel como desecante y se almacenaron entre $2^{\circ} \mathrm{C}$ y $8^{\circ} \mathrm{C}$ hasta su análisis.

\section{Estudio de las características de interés.}

Con el objetivo de determinar la concentración de los controles de T4,TSH y Phe en los diferentes tipos de papel de filtro se realizaron 20 determinaciones para cada control durante 5 días consecutivos para un total de 100 determinaciones. El cálculo de la concentración promedio de cada control, el coeficiente de variación y la prueba t de "student's" para datos pareados se realizó a través de las funciones para análisis estadístico contenida en la hoja de cálculo de Microsoft Excel 2000.

Se realizó un estudio de estabilidad en el tiempo; a los 3, 6 y 9 meses, para los controles en papeles de filtro $\mathbf{S} \& \mathbf{S} 2992$ y $\mathbf{S} \& \mathbf{S} 903$ conservados entre $2^{\circ} \mathrm{C}$ 


\section{A Frómeta-Suárez, N Marrero-González, E González-Reyes, E Lugo-Vallejo y col.}

y $8^{\circ} \mathrm{C}$ y se determinó el porcentaje de pérdida de actividad de los analitos estudiados a tiempo real.

Se evaluaron 400 muestras de sangre de talón de neonatos colectadas simultáneamente en papel de filtro $\mathbf{S} \& \mathbf{S} 2992$ y $\mathbf{S} \& \mathbf{S} 903^{\mathrm{TM}}$ procedentes del programa nacional cubano de detección de Fenilcetonuria.

\section{Métodos.}

Para realizar las mediciones de T4 total, TSH y Phe se emplearon los estuches de reactivos: UMELISA TSH Neonatal, UMELISA T4 Neonatal y el UMTEST PKU respectivamente; producidos por el Centro de Inmunoensayo (7-10).

Los calibradores y controles empleados fueron preparados a partir de sangre total humana ajustada a un valor de hematocrito de 55\% y dispensados sobre papel de filtro Schleicher \& Schuell 2992, Schleicher $\&$ Schuell $903^{\mathrm{TM}}$ y Whatman BFC 180. Se emplearon discos de $5 \mathrm{~mm}$ de diámetro de los calibradores y controles.

El UMELISA TSH Neonatal es un ensayo inmunoenzimático heterogéneo tipo sandwich, en el cual se utiliza como fase sólida tiras de ultramicroelisa (10 $\mu \mathrm{L}$ por pocillo) revestidas previamente con anticuerpos monoclonales anti cadena $\beta$ de la TSH. Las muestras se eluyen con $70 \mu \mathrm{L}$ de conjugado anti $\alpha$ TSH/Fosfatasa Alcalina en solución Tampón TRIScarnero, luego se depositan en las tiras de reacción y finalmente se añade el sustrato fluorigénico (4Metilumbeliferil fosfato). La curva estándar fue validada contra el patrón 80558 de la OMS, abarca un rango útil de 10-200 mUI/L, con un límite de detección de $2 \mathrm{mUI} / \mathrm{L}$. La evaluación analítica mostró una precisión intra e inter-ensayo de 6.2 y $7.4 \%$ respectivamente. El porcentaje de recuperación obtenido al añadir la hormona fue de 96 a 100\% .

El UMELISA T4 Neonatal es un ensayo heterogéneo inmunoenzimático competitivo, en el cual se utiliza como fase sólida tiras de ultramicroelisa (10 $\mu \mathrm{L}$ por pocillo) revestidas previamente con anticuerpos anti T4. Las muestras se eluyen con $70 \mu \mathrm{L}$ de conjugado T4/Fosfatasa Alcalina en solución Tampón Barbiturato- ANS, luego se depositan en las tiras de reacción y finalmente se añade el sustrato fluorigénico (4- Metilumbeliferil fosfato). La curva estándar fue preparada por el método gravimétrico y abarca un rango útil de $25-400 \mathrm{nmol} / \mathrm{L}$, con un límite de detección de $11 \mathrm{nmol} / \mathrm{L}$. La evaluación analítica mostró una precisión intra e inter-ensayo de 6.9 y $9.1 \%$ respectivamente. La recuperación analítica obtenida fue mayor del $95 \%$ y no se evidenciaron interferencias significativas con otras sustancias relacionadas estructuralmente.

El UMTEST PKU es una prueba fluorescente basada en el método de McCaman \& Robins (11), en esta prueba la Phe presente en la muestra se eluye con $70 \mu \mathrm{L}$ de etanol al $70 \%$ y luego reacciona con la ninhidrina, en condiciones óptimas de $\mathrm{pH}$ y temperatura, formando un complejo poco fluorescente. Con la adición de iones cobre, se produce la amplificación de la fluorescencia, aumentando su intensidad por la previa adición de LLeucil-L-Alanina a la mezcla de reacción. La curva estándar fue preparada por el método gravimétrico y abarca un rango útil de $180-2880 \mu \mathrm{mol} / \mathrm{L}$, con un límite de detección de $50 \mu \mathrm{mol} / \mathrm{L}$. La evaluación analítica mostró una precisión intra e inter-ensayo de 3.8 y 5.2\% respectivamente. El porcentaje de recuperación obtenido fue de 94 a $115 \%$.

La medición de la señal de fluorescencia se realizó en todos los casos, empleando el lector FluorímetroFotómetro PR-521 (rango espectral de fluorescencia: $420-500 \mathrm{~nm}$ ) y los resultados fueron calculados automáticamente empleando el Strips reader software Ver.7.0 soportado sobre Windows, un programa diseñado para el cálculo de la concentración e interpretación de los resultados. Otros equipos y accesorios empleados fueron el lavador automático de placas MAS $301 \mathrm{y}$ el perforador de discos de papel de $5 \mathrm{~mm}$ de diámetro P-51.

\section{RESULTADOS Y DISCUSIÓN.}

Se realizó la medición de las concentraciones correspondientes a cada uno de los controles para TSH, T4 y Phe.

Los datos de los cuadros 1,2 y 3 muestran coeficientes de variación inferiores al $11 \%$ en el papel

\section{Revista Biomédica}


Papeles de filtro en los ensayos UMELISA.

Cuadro 1

Comparación entre los papeles de filtro Whatman BFC 180 y el $S \& S 903^{\mathrm{TM}}$ usando como referencia el S\&S 2992 para los controles de TSH.

\begin{tabular}{|c|c|c|c|c|c|}
\hline \multicolumn{2}{|c|}{$\begin{array}{l}\text { Whatman } \\
\text { BFC } 180\end{array}$} & \multicolumn{2}{|c|}{$\begin{array}{l}\text { S\&S } \\
903^{\mathrm{TM}}\end{array}$} & \multicolumn{2}{|c|}{$\begin{array}{l}\text { S\&S } \\
2992\end{array}$} \\
\hline $\mathbf{C}_{\mathbf{p}}$ & CV & $\mathbf{C}_{\mathbf{p}}$ & CV & $\mathrm{C}_{\mathrm{p}}$ & CV \\
\hline 11.1 & 2.6 & 11.7 & 6.9 & 11.3 & 6.8 \\
\hline 21.1 & 10.7 & 22.3 & 8.1 & 21.7 & 9.3 \\
\hline 33.5 & 4.4 & 32.6 & 4.4 & 32.9 & 1.9 \\
\hline 63.8 & 5.4 & 61.3 & 6.4 & 60.5 & 6.0 \\
\hline
\end{tabular}

$\mathrm{C}_{\mathrm{p}}$ : Concentración promedio (mUI/L) CV: Coeficiente de Variación $(\%)$.

Whatman BFC 180 e inferiores a $10.6 \%$ en papel $\mathbf{S} \& \mathbf{S}$ $903^{\mathrm{TM}}$ para los diferentes analitos estudiados; estos resultados revelan que ambos papeles de filtro pueden ser utilizados en la colecta de las muestras y en la preparación de los calibradores y controles de los UMELISA T4 y TSH Neonatal y el UMTEST PKU, pues no producen afectación en la precisión y exactitud de estos métodos, los cuales fueron estandarizados originalmente utilizando el papel S\&S 2992.

La aplicación de la prueba t de "Student's" para datos pareados demostró que no existen diferencias estadísticamente significativas entre ambos tipos de papel de filtro para un intervalo de confianza del $95 \%$

\section{Cuadro 2}

Comparación entre los papeles de filtro Whatman BFC 180 y el $S \& S 903^{\mathrm{TM}}$ usando como referencia el $S \& S 2992$ para los controles de $\mathrm{T} 4$.

\begin{tabular}{clllll}
\hline \multicolumn{2}{c}{ Whatman } & \multicolumn{2}{c}{ S\&S } & \multicolumn{2}{c}{ S\&S } \\
\multicolumn{2}{c}{ BFC 180 } & \multicolumn{2}{c}{$903^{\mathrm{TM}}$} & \multicolumn{2}{c}{2992} \\
$\mathrm{C}_{\mathrm{p}}$ & $\mathrm{CV}$ & $\mathrm{C}_{\mathrm{p}}$ & $\mathrm{CV}$ & $\mathrm{C}_{\mathrm{p}}$ & $\mathrm{CV}$ \\
\hline $\mathbf{8 2 . 2}$ & $\mathbf{7 . 8}$ & $\mathbf{8 7 . 7}$ & $\mathbf{7 . 4}$ & $\mathbf{7 8 . 3}$ & $\mathbf{2 . 5}$ \\
$\mathbf{1 3 1 . 2}$ & $\mathbf{8 . 0}$ & $\mathbf{1 3 6 . 4}$ & $\mathbf{2 . 5}$ & $\mathbf{1 1 8 . 8}$ & $\mathbf{6 . 1}$ \\
$\mathbf{1 8 8 . 6}$ & $\mathbf{2 . 0}$ & $\mathbf{2 0 0 . 1}$ & $\mathbf{1 0 . 6}$ & $\mathbf{1 8 5 . 3}$ & $\mathbf{1 2 . 8}$ \\
$\mathbf{3 2 8 . 7}$ & $\mathbf{1 0 . 9}$ & $\mathbf{2 9 6 . 9}$ & $\mathbf{6 . 9}$ & $\mathbf{3 0 4 . 4}$ & $\mathbf{8 . 5}$ \\
\hline
\end{tabular}

$$
p=0.10 \quad p=0.22
$$

$\mathrm{C}_{\mathrm{p}}$ : Concentración promedio (nmol/L) CV: Coeficiente de Variación $(\%)$

\section{Cuadro 3}

Comparación entre los papeles de filtro Whatman BFC 180 y el $S \& S 903^{\mathrm{TM}}$ usando como referencia el $\mathrm{S} \& S 2992$ para los controles de Phe.

\begin{tabular}{cccccc}
\hline \multicolumn{2}{c}{ Whatman } & \multicolumn{2}{c}{ S\&S } & \multicolumn{2}{c}{ S\&S } \\
\multicolumn{2}{c}{ BFC 180 $^{2}$} & \multicolumn{2}{c}{$903^{\mathrm{TM}}$} & \multicolumn{2}{c}{2992} \\
$\mathrm{C}_{\mathrm{p}}$ & $\mathrm{CV}$ & $\mathrm{C}_{\mathrm{p}}$ & $\mathrm{CV}$ & $\mathrm{C}_{\mathrm{p}}$ & $\mathrm{CV}$ \\
\hline 2.9 & $\mathbf{4 . 8}$ & $\mathbf{2 . 8}$ & $\mathbf{5 . 7}$ & $\mathbf{2 . 7}$ & $\mathbf{3 . 2}$ \\
4.2 & $\mathbf{9 . 7}$ & $\mathbf{4 . 9}$ & $\mathbf{9 . 9}$ & $\mathbf{4 . 5}$ & $\mathbf{6 . 1}$ \\
$\mathbf{5 . 9}$ & $\mathbf{8 . 2}$ & $\mathbf{6 . 2}$ & $\mathbf{5 . 4}$ & $\mathbf{5 . 8}$ & $\mathbf{8 . 6}$ \\
$\mathbf{9 . 6}$ & $\mathbf{3 . 8}$ & $\mathbf{1 0 . 0}$ & $\mathbf{8 . 8}$ & $\mathbf{9 . 6}$ & $\mathbf{5 . 9}$ \\
\hline \multicolumn{2}{c}{$\mathrm{p}=\mathbf{0 . 0 8}$} & \multicolumn{3}{c}{$\mathrm{p}=\mathbf{0 . 0 8}$} \\
\end{tabular}

$\mathrm{C}_{\mathrm{p}}$ : Concentración promedio $(\mathrm{mg} / \mathrm{dL}) \quad \mathrm{CV}$ : Coeficiente de Variación (\%)

al compararlos con el S\&S 2992. Resultados similares obtuvieron Marsden y colaboradores (12) al hacer un estudio comparativo de los papeles de filtro Whatman BFC 180 y $\mathbf{S} \& \mathbf{S} 903^{\mathrm{TM}}$ utilizados en los ensayos para el tamizaje neonatal de la tripsina inmunoreactiva, galactosa y 17- hidroxiprogesterona.

\section{Estudio de Estabilidad.}

El tiempo que media entre el momento de colección de las muestras y su análisis en el laboratorio, las diferentes condiciones de temperatura a las cuales están sometidas durante su transportación y almacenamiento pudiera comprometer los resultados analíticos de los diferentes ensayos, es por ello que consideramos útil para nuestro estudio evaluar la estabilidad de la tiroxina, tirotropina y la fenilalanina durante un período de 9 meses. La estabilidad de un analito es comúnmente tomada como el tiempo durante el cual ocurre un cambio del $10 \%$ de su concentración con respecto a la concentración original (13). Pero consideraremos como válido, para nuestro propósito, un 20\% de pérdida de actividad valor con el cual la inexactitud de una muestra es de un $10 \%$ de variación.

Los resultados obtenidos (figura 1), al evaluar el porcentaje de pérdida de actividad de los controles en los papeles S\&S 2992 y $903^{\mathrm{TM}}$ a los 3; 6 y 9 meses fueron inferiores al 17\% para TSH y $13 \%$ para T4, para los controles de Phe el porcentaje de pérdida de actividad fue inferior al 6.5\%. La pobre recuperación

Vol.13/No.4/Octubre-Diciembre, 2002 
A Frómeta-Suárez, N Marrero-González, E González-Reyes, E Lugo-Vallejo y col.

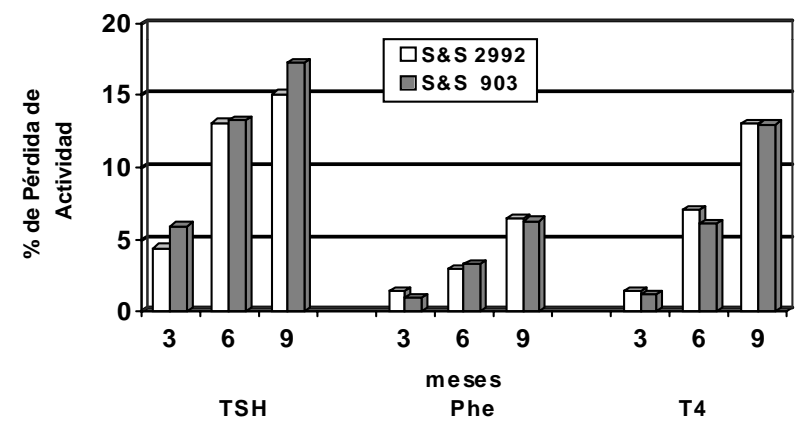

Figura 1.- Estabilidad de los controles de TSH, T4 y Phe en papel S\&S 2992 y S\&S 903 ${ }^{\mathrm{TM}}$.

de ciertos metabolitos en sangre seca sobre papel de filtro está probablemente relacionada con reacciones químicas no catalíticas más que con procesos biológicos en sí, la composición de la muestra puede cambiar si se toma en cuenta que los microorganismos pudieran tener un caldo de cultivo perfecto al estar alimentándose de los componentes de esta y excretando lo que su metabolismo determina. Marrero y colaboradores (14) observaron una pérdida promedio de actividad de T4 de $19 \%$, en muestras conservadas a $28^{\circ} \mathrm{C}$ y con una humedad relativa del 88\%; sin embargo Harvey y colaboradores (15) realizaron estudios acerca de la estabilidad de la fenilalanina, galactosa y otros aminoácidos y encontraron que la fenilalanina en muestras de sangre en papel de filtro conservadas a temperatura ambiente, es estable por un período de tiempo de hasta 16 años. Las muestras en papel de filtro colectadas para el tamizaje neonatal constituyen una fuente valiosa de información ya que se puede

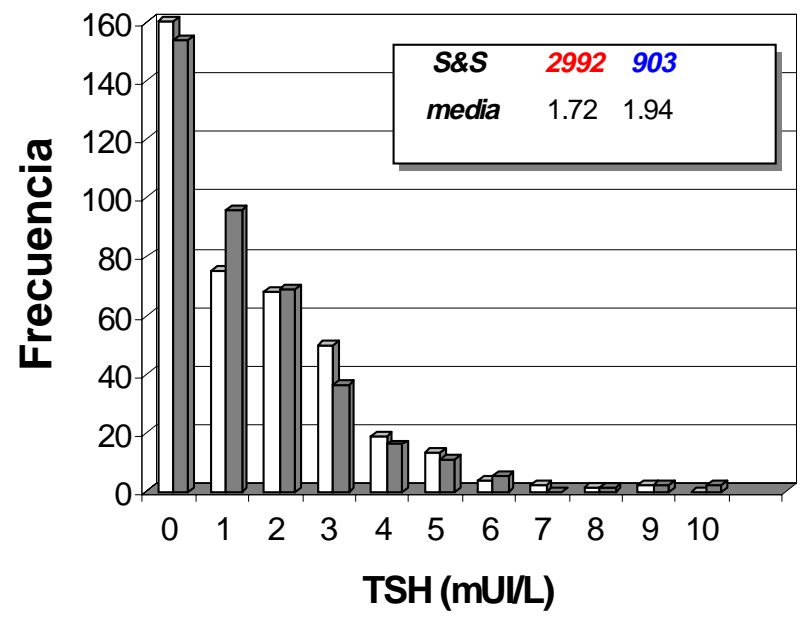

F'igura 2.- Distribución de frecuencias de la concentración de TSH en papel S\&S 2992 y S\&S 903 ${ }^{\mathrm{TM}}$.

Revista Biomédica

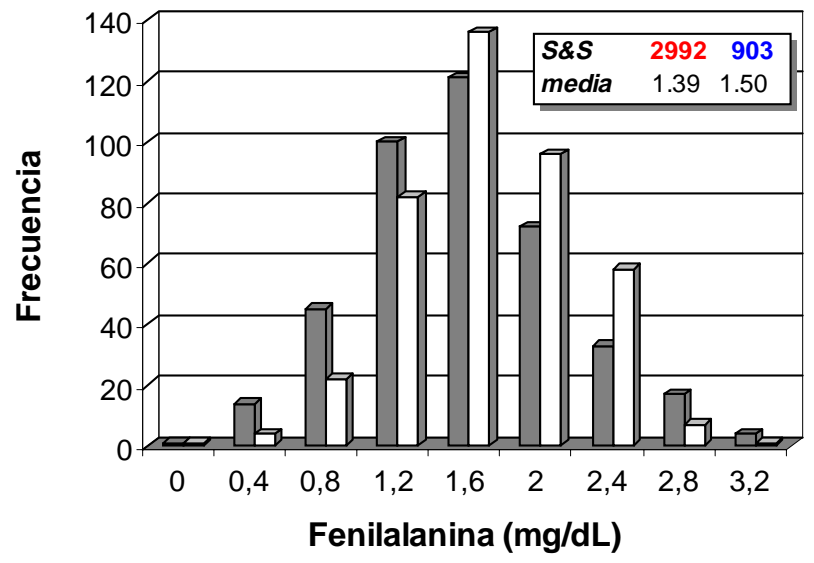

Figura 3.- Distribución de frecuencias de la concentración de Phe en papel S\&S 2992 y S\&S 903 ${ }^{\mathrm{TM}}$.

conocer la expresión temprana de ciertos desórdenes metabólicos en el neonato y siempre que sea posible estas deben ser conservadas por varios años; sin embargo la confiabilidad de la información depende del conocimiento de la estabilidad de la sustancia en las muestras almacenadas (16).

Como resultado de una encuesta a la comunidad internacional que se dedica al tamizaje neonatal y con el objetivo de mejorar la exactitud de los ensayos analíticos, de homogeneizar los controles y materiales de referencia, la compañía Schleicher \& Schuell tomó la decisión de comercializar solamente el papel de filtro S\&S 903 ${ }^{\mathrm{TM}}$ para su uso en los programas de tamiz neonatal. En nuestro país los programas nacionales para la detección de errores innatos del metabolismo se llevan a cabo utilizando muestras colectadas en papel S\&S 2992 y por tanto decidimos evaluar la concentración de fenilalanina y TSH en 400 muestras colectadas simultáneamente en papel S\&S 2992 y $\mathbf{S} \& \mathbf{S}$ 903 ${ }^{\mathrm{TM}}$.

La cuantificación de los analitos se realizó en el UMTEST PKU y en el UMELISA TSH Neonatal, en las figuras 2 y 3 se muestra la distribución de frecuencias de la concentración de TSH y Phe respectivamente. Los valores promedios de concentración en ambas distribuciones son ligeramente mayores en las muestras colectadas en papel $903^{\mathrm{TM}}$, así como, el patrón de distribución de frecuencias de ambos analitos está levemente desplazado hacia la derecha, reportes de Dhondt y colaboradores(17) confirman que a través 


\section{Papeles de filtro en los ensayos UMELISA.}

de un estudio realizado en calibradores de fenilalanina la capacidad de absorción del papel $\mathbf{S} \& \mathbf{S} 903^{\mathrm{TM}}$ es 1.1 mayor que la del $\mathbf{S} \& \mathbf{S} 2992$. Esta diferencia se acentúa un poco más si los estuches de reactivos que se utilizan en los laboratorios de tamiz neonatal tienen calibradores en papel $903^{\mathrm{TM}}$ y las muestras se colectan en papel 2992. De no tomarse en cuenta este aspecto se pueden encontrar valores de concentración discordantes entre un 10-20\% más bajos (4), lo cual influye en la efectividad de los programas de tamizaje neonatal.

\section{REFERENCIAS.}

1.- Fisher DA. Screening for congenital hypothyroidism. TEM 1991; 2 : 129-33.

2.- Partnering for quality results. S\&S 903 paper is the international standard for specimen collection, transport and archiving. Disponible en: http://www.s-and-s.com.

3.- Therrell BL, Hannon HW, Kenneth AP, Lorey F, Brokopp $\mathrm{C}$, Eckman J, et al. Guidelines for the retention, storage and use of residual dried blood spot samples after newborn screening analysis: Statement of the council of regional networks for genetic services. Biochem Mol Med 1996; 57:116-24.

4.- Elvers LH, Loeber JG. The need for standardized bloodspot TSH-calibrators in congenital hypothyroidism screening. Early Human Development 1996; 45:179-90.

5.- Addisson GM. How should screening programs choose the optimum collection paper. The UK experiencie (version on CD-ROM). EG \& G Wallac producers. ISNS Quality assurance and standardization meeting. June 11-12;1999.

6.- National Committee for Clinical Laboratory Standards (NCCLS). Proposed guideline. Calibration and control materials for neonatal hypothyroid screening programs. NCCLS 1990; $3: 225-45$.

7.- Frómeta A, Lechuga MF, Pérez PL, Marrero N, Urquiza HD, Coto R, et al. Desenvolvimento do UMELISA TSH Neonatal para dosagem de TSH em sangue coletado em papel filtro. Rev Brasil Anal Clin 1996; 28: 202-4.

8.- Frómeta A, Lechuga MF, Pérez PL, Marrero N, Solís RL, Robaina R, et al. Development of an UMELISA TSH Neonatal for the quantification of thyroid stimulating hormone (TSH) in blood spot collected on filter paper. In: Levy HL, Hermos RJ, Grady GF, editors. Proceedings of third meeting of the international society for neonatal screening; 1996 Oct 20-23; Boston, EUA. Massachusetts: IKON/MAP; 1996. p. 238-9.

9.- Almenares P, Lechuga MF, Marrero N, Solís RL, Frómeta A, Robaina R, et al. Development of UMELISA T4 Neonatal for the early diagnosis of congenital hypothyroidism. In: Levy HL, Hermos RJ, Grady GF, editors. Proceedings of third meeting of the international society for neonatal screening; 1996 Oct 20-23; Boston, EUA. Massachusetts: IKON/MAP; 1996. p. 240-1.

10.- Machado C, Solís RL, Bécquer D, Cazanave J, Fernández JL. The ultramicroanalytical system (SUMA) as a new approach to the newborn screening for hyperphenylalaninemias. In: Levy HL, Hermos RJ, Grady GF, editors. Proceedings of third meeting of the international society for neonatal screening; 1996 Oct 20-23; Boston, EUA. Massachusetts: IKON/MAP; 1996. p. 238-9.

11.- McCaman MW , Robins E. Fluorimetric method for the determination of phenylalanine in serum. J Lab Clin Med 1962; 59:885-90.

12.- Marsden AM, Pasquet-Ferré C. The Comparative performances of Whatman BFC 180 and S\&S 903 filter papers in newborn Screening Assays for IRT, 17-OH and Galactose. In: Levy HL, Hermos RJ, Grady GF, editors. Proceedings of the third meeting of the international society for neonatal screening; 1996 Oct. 20-23; Boston, EUA. Massachusetts: IKON/MAP; 1996. p.102-4.

13.- Alonso- Fernández JR. Cobertura de los programas de Tría Neonatal en España y estrategias de toma de muestra. Prevención de Enfermedades Metabólicas Congénitas 1993; 6:5-8.

14.- Marrero N, González Y, Frómeta A, Lechuga MF. Estabilidade de tiroxina (T4) em manchas de sangue seco sobre papel de filtro. Impacto na pesquisa neonatal de hipotireoidismo congénito. NewsLab 1998; 26:50-60.

15.- Levy HL, Simmons JR, McCready RA. Stability of amino acids and galactose in the newborn screening filter paper blood specimen. J Pediatr 1985; 107: 757-60.

16.- Dezateux C. Evaluating newborn screening programmes based on dried blood spots: future challenges. Br Med Bull 1998; 54 (4):877-90.

17.- Dhondt JL, Paux E, Farriaux JP. New for a standardized procedure in the preparation of phenylalanine calibrators. Early Human Development 1996; 45:277-85.

Vol.13/No.4/Octubre-Diciembre, 2002 